1 Universidade Federal de São Paulo (Unifesp) - São Paulo (SP), Brasil.

leonardo.carnut@gmail.com

2 Universidade de São Paulo (USP) - São Paulo (SP), Brasil.

3 Pontifícia Universidade Católica de São Paulo (PUC-SP) - São Paulo (SP) Brasil.

\section{Metodologias para alocação equitativa de recursos financeiros em saúde: uma revisão integrativa}

\author{
Methodologies for equitable allocation of health financial resources: \\ an integrative review
}

Leonardo Carnut', Áquilas Mendes $\mathbf{2 , 3}$, Marcel Guedes Leite ${ }^{\mathbf{3}}$
RESUMO Há diversas experiências no mundo que visam ao desenvolvimento de metodologias para alocação de recursos para a saúde, contudo, o que resta saber é até que ponto a equidade é operacionalizada nesses métodos. Por isso, este estudo objetivou analisar o que tem sido produzido nas literaturas nacional e internacional a respeito das metodologias de alocação equitativas de recursos em saúde e suas dimensões. Realizou-se uma revisão integrativa em três portais/bases de dados (Bireme, PubMed e Scopus) de artigos científicos, publicados em português, inglês e espanhol. Foram identificados nos artigos o(s) objetivo(s), o método do estudo utilizado pelos pesquisadores e a abordagem sobre a metodologia de alocação de recursos em saúde no que se refere à discussão/operacionalização da equidade. Ainda são poucos os estudos em que alocação equitativa é tema central. Há certa imprecisão sobre a delimitação entre 'alocar' e 'financiar'. Em geral, as metodologias precisam admitir as implicações (bio)éticas relativas à equidade, devem se basear minimamente na dimensão per capita, em conjunto, compulsoriamente, com a orçamentação incremental, com as questões sociodemográficas, sociossanitárias e epidemiológicas e ter centralidade na 'necessidade de saúde', sendo necessário um constante aperfeiçoamento da metodologia ao longo do tempo para refinar a operacionalidade da equidade.

PALAVRAS-CHAVE Alocação de recursos. Financiamento governamental. Sistemas de saúde. Financiamento da assistência à saúde. Literatura de revisão como assunto.

\begin{abstract}
There are several experiences in the world that aim to develop methodologies for resource allocation for health, however, what remains to be seen is the extent to which equity is operationalized in these methods. Therefore, this study aimed to analyze what has been produced in national and international literature regarding equitable methodologies for the allocation of health resources and their dimensions. An integrative review was conducted in three portals/databases (Bireme, PubMed and Scopus) of scientific articles, published in Portuguese, English and Spanish. The articles identified the objective(s), the study method used by the researchers and the approach on the methodology of health resource allocation with regard to the discussion/operationalization of equity. There are still few studies in which equitable allocation is the central theme. There is some vagueness about the boundary between 'allocate' and 'finance'. In general, methodologies need to admit the (bio)ethical implications related to equity, they must be based minimally on the per capita dimension, together, mandatorily, with incremental budgeting, sociodemographic, sociosanitary and epidemiological issues and centered on the 'health needs', requiring a constant refinement of the methodology over time to refine the operability of equity.
\end{abstract}

KEYWORDS Resource allocation. Government financing. Health systems. Healthcare financing. Review literature as topic. 


\section{Introdução}

O direito à saúde universal, integral e gratuito, embora positivado pela Constituição Federal de 1988', em seus artigos de 196 a 200, apresenta diversos desafios no que se refere à sua efetivação. Um desses desafios no alcance do princípio da equidade é, sem dúvida, garantir a alocação de recursos do nível federal aos entes subnacionais. Com vistas a alcançar a equidade, é pertinente considerar as dimensões continentais do Brasil, a complexa relação entre os três níveis da federação e a profunda desigualdade de renda, que se manifesta tanto nas classes sociais quanto entre as várias regiões.

No entanto, ao compreender como se consolidou a alocação de recursos em saúde ao longo da história do Sistema Único de Saúde (SUS), precisa-se recorrer ao contexto do subfinanciamento desse sistema, pois a equidade na alocação ganha ainda mais relevância nessa situação. Isso porque os recursos do SUS já partem de um subfinanciamento crônico desde os anos de 1990, e, por isso, Mendes² e OckéReis, Ribeiro e Piola ${ }^{3}$ já denunciavam que os recursos do Orçamento da Seguridade Social deveriam, nos anos subsequentes pós-constituinte, ser repartidos entre as três políticas sociais (saúde, previdência e assistência social) que compõem o bloco da seguridade. Contudo, tais recursos nunca foram garantidos à saúde pública brasileira.

Assim, ao contrário do que determina a Carta Magna, os recursos para garantir o financiamento e o pleno funcionamento do SUS foram sendo, ao longo do tempo, restringidos por normas infraconstitucionais ${ }^{2}$. Fernando Henrique Cardoso, em 1993, asfixiou os repasses para a saúde através da criação do Fundo Social de Emergência (FSE), que, logo em seguida, passou a ser chamado de Desvinculação das Receitas da União (DRU), diminuindo a quantidade de recursos vinculados ao orçamento público em $20 \%{ }^{4}$. Recentemente, após a proposição do Governo Dilma, sendo promulgado pelo Governo Temer, 30\% desse valor já é desvinculado desse orçamento, conforme a Emenda Constitucional $n^{\circ}$ 93/2016 .

Contudo, apenas no governo Dilma, após o estabelecido pelo artigo $n^{\circ} 35$ da Lei Orgânica da Saúde no 8.080/1990, ocorre a instituição de critérios de alocação dos recursos federais aos entes subnacionais baseados na dimensão necessidades de saúde, com a publicação da Lei Complementar $\mathrm{n}^{\circ} 141$ (LC-141), em $2012^{6}$. Esta se referia a uma pauta histórica de luta dos movimentos sociais e de gestores do sistema para a regulamentação da Emenda Constitucional 29 (EC-29).

Nessa lei, três elementos são aqueles que definem a alocação dos poucos recursos nessa área: (a) a definição do que são as Ações e os Serviços Públicos de Saúde (ASPS); (b) os percentuais mínimos para aplicação da União, estados e municípios em saúde; e (c) as regras (critérios) para transferências de recursos para financiamento da saúde entre os entes federados. Assim, o art. 17 da Lei n ${ }^{0} 141$ é claro:

Art. 17. O rateio dos recursos da União vinculados a ações e serviços públicos de saúde [...] observará as 'necessidades de saúde' da população, 'as dimensões epidemiológica, demográfica, socioeconômica, espacial' e de 'capacidade de oferta' de 'ações e de serviços de saúde' e, ainda, o disposto no art. 35 da Lei no 8.080 , de 19 de setembro de 1990, de forma a atender os objetivos do inciso II do § 3으 do art. 198 da Constituição Federal. [grifo nosso] ${ }^{6}$.

Ressalte-se, neste ponto, que a LC-141 determina que o governo federal estabeleça e torne pública, todos os anos, uma 'metodologia' para que seja realizada a alocação dos recursos financeiros federais para financiamento de ações e serviços públicos de saúde a estados e municípios. Tal metodologia deve considerar todos os critérios descritos no art. 17 (entre aspas simples), e conforme determina, inclusive, o artigo 35 da Lei Orgânica da Saúde no 8.080/1990. 
No entanto, fica a dúvida sobre como operacionalizar tais critérios, haja vista que eleger indicadores, utilizar os melhores do ponto de vista metodológico e ainda articulá-los na tentativa de operacionalizar a equidade não é uma tarefa trivial. Isso se deve ao fato de que o próprio conceito de equidade, mesmo tendo de ser perseguido do ponto de vista prático, é um desafio em si mesmo, conforme já descrito por Porto et al. ${ }^{7}$, quando a autora criticava a malversação do termo ao discorrer sobre a equidade como a mera distribuição igualitária de recursos.

Conforme nos ensinam Mendes, Leite e Marques $^{8}$, há diversas experiências no mundo que visam ao desenvolvimento de metodologias para alocação de recursos para a saúde. Nesse sentido, para que a distribuição dos recursos dos poderes centrais para os entes subnacionais seja realizada de forma 'equitativa', ela deve levar em conta critérios que abordem as necessidades de saúde da população e que devem ser revisados constantemente ${ }^{7}$ para sua atualização, considerando-se que as necessidades são dinâmicas.

Contudo, o debate reaparece quando se tenta conhecer metodologias na literatura nacional e internacional que operem essa alocação com indicadores que componham o sentido do termo 'equidade', isto é, alocar mais recursos àqueles entes que mais precisam e menos recursos aos entes que menos precisam ${ }^{8}$.

Assim, compreendendo que este é um debate em aberto, o presente artigo tem o intuito de realizar uma revisão integrativa, a fim de analisar o que tem sido produzido nas literaturas nacional e internacional a respeito das metodologias de alocação equitativas de recursos em saúde e suas dimensões, que, no Brasil, ganharam o nome genérico de 'critérios de rateio' para alocar recursos do nível federal para os entes subnacionais no SUS.

\section{Material e métodos}

Realizou-se uma revisão integrativa conforme apontado por Soares et al. ${ }^{9}$. Nesse contexto, este estudo enquadra-se na definição de 1998 recuperada pelas autoras em que as revisões integrativas são relatórios integrados dos conceitos-chave identificados nos estudos que se analisam. A literatura pesquisada abrange todos os estudos que apresentam hipóteses idênticas ou relacionadas à da revisão e deve ser tão rigorosa quanto a dos estudos primários, com aplicação cuidadosa de critérios de avaliação, para minimizar os efeitos da subjetividade.

Assim sendo, para dar continuidade ao processo de revisão, procedeu-se à identificação dos descritores em língua portuguesa, por meio da base de dados dos Descritores em Ciências da Saúde (DeCS), a partir dos itens-chave da pergunta de pesquisa, qual seja: 'O que há na produção literária nacional e internacional a respeito de experiências governamentais sobre metodologias para alocação de recursos financeiros em sistemas de saúde?'.

Tendo em vista a necessidade de levantamento de produção acadêmica para além das fronteiras da América Latina, sobretudo pelo fato de os sistemas de saúde universais mais bem estabelecidos se encontrarem no continente europeu, foi identificada a correspondência desses descritores em língua inglesa, por meio da utilização dos chamados $\mathrm{MeSH}$ Terms, no portal PubMed.

Aqui cabe uma importante observação a respeito de não estarem presentes nas buscas alguns descritores que sejam compostos pelo termo 'equidade'. Isso não invalida a escolha dos descritores utilizados nas sintaxes finais, haja vista que, ao ler o conteúdo dos descritores, a ideia de equidade estava ali presente.

Após a definição dos dois grupos de descritores, foram iniciadas buscas exploratórias em dois portais de bases de dados: Centro Latino-Americano e do Caribe de Informação em Ciências da Saúde (Bireme) e PubMed, e uma base de dados específica: a Scopus. Combinando-se os descritores por meio dos operadores booleanos $A N D$ e $O R$, foi possível chegar às sintaxes finais para cada base/portais (quadro 1), que apresentaram resultados 
satisfatórios para responder à indagação motivadora deste artigo, assim como definir as principais variáveis (polos) de interesse da presente pesquisa, quais sejam: (a) alocação de recursos em saúde, (b) financiamento da saúde e (c) governo.

Quadro 1. Base/Portais de dados, sintaxe final e número de estudos identificados que potencialmente respondiam à pergunta da revisão. 2019*

\begin{tabular}{lll}
\hline Base/Portais & Sintaxe final & $\begin{array}{c}\text { Número de estudos } \\
\text { identificados }\end{array}$ \\
\hline Bireme & (mh:("ALOCACAO de recursos" or "ALOCACAO de recursos em & 182 \\
& saude" or "ALOCACAO de recursos para a atencao a saude")) AND & \\
& (mh:("FINANCIAMENTO" or "organizacao do FINANCIAMENTO" or \\
& "FINANCIAMENTO da saude" or "FINANCIAMENTO governamental" or \\
& "FINANCIAMENTO publico")) AND (mh:("federalismo" or "GOVERNO do \\
& municipio" or "GOVERNO estadual" or "GOVERNO federal" or "GOVERNO \\
& LOCAL" or "regionalizacao") or ("sistemas locais de saude" or "SISTEMAS \\
& DE SAUDE" or "descentralizacao")) \\
& (((Health Resources[MeSH Terms] OR Resource Allocation[MeSH Terms] & 123 \\
& OR Financing, Government[MeSH Terms] OR Health Care Rationing[MeSH \\
& Terms] OR Financial resources in health[MeSH Terms])) AND Local \\
& Government[MeSH Terms]) AND (Public Assistance[MeSH Terms] OR \\
& universal coverage[MeSH Terms] OR National Health Programs[MeSH \\
& Terms]) \\
& KEY ("health care rationing") OR KEY ("resource allocation") OR KEY \\
& ("equity in resource allocation") OR KEY ("health resources") OR KEY \\
& ("financial resources in health") AND KEY ("Financing, Government") \\
& OR KEY ("Local Government") AND KEY ("Public Assistance") OR KEY \\
& ("Universal Coverage") OR KEY ("National Health Programs") OR KEY \\
& ("health systems") \\
\hline Scopus & \\
\hline
\end{tabular}

${ }^{\star}$ A busca dessas sintaxes finais foi realizada em março de 2019. É possível que esse número tenha aumentado, seja pela incorporação de novas bases de dados (no caso do portal Bireme) ou por novos estudos indexados com os descritores acima após março de 2019.

Em seguida, foi realizada leitura sistematizada dos títulos e resumos dos resultados encontrados nas buscas das três plataformas (base/portais), incluindo na seleção para análise apenas artigos científicos, publicados em português, inglês e espanhol, e que abordassem a alocação de recursos financeiros em saúde. Dessa maneira, foram excluídas as produções acadêmicas, como monografias e teses, além de anais de congressos e publicações oficiais. Na subsequente categorização dos resultados das buscas, foram identificadas e excluídas aquelas produções que (a) não tratam da temática geral deste estudo ou (b) tratam da temática, mas não respondem à pergunta da pesquisa. Por fim, chegou-se ao número final de 19 artigos a serem analisados neste estudo que foram considerados como estudos incluídos na revisão (artigos incluídos).

É importante ressaltar que nesta revisão optou-se por focar apenas em estudos primários, portanto, não incluindo revisões já realizadas sobre esse assunto. Neste sentido, não se desejou realizar uma 'revisão de revisões', isto é, uma metarrevisão. Em que pese que esta revisão apresenta limites, como todo e qualquer método o tem, reconhece-se que, além de recuperar artigos internacionais, ela possibilitou recuperar, também, artigos nacionais, fundamentais para a discussão da 
realidade brasileira. Isso pode ser considerado um avanço no debate, pois, ao encontrar essas experiências, evita-se, assim, certo colonialismo da discussão internacional sobre o assunto quando se trata de SUS.

\section{Resultados}

A maior parte dos resultados encontrados nas sintaxes finais compreendeu publicações e materiais em línguas inglesa, espanhola e portuguesa, havendo poucos casos de publicações em chinês, em russo e em línguas nórdicas, como sueco e norueguês, em cujo caso as publicações foram excluídas.

Outro importante achado é o fato de que, aproximadamente, $85 \%$ dos resultados remanescentes das buscas, depois do filtro dos idiomas, não tratam diretamente sobre a temática de metodologias de alocação equitativa de recursos. De fato, muitos dos artigos e teses excluídos nessa etapa tratavam de algum aspecto do financiamento do setor saúde, de gestão de recursos públicos, de reformas e políticas de determinados sistemas de saúde, de bioética e de priorização de tipos de cuidado. Contudo, não tratavam, nem tangencialmente, do tema desta revisão.

Ainda com relação aos estudos excluídos, destaca-se que pouco menos de $80 \%$ dos artigos e publicações remanescentes, muito embora tratem da temática sobre a alocação de recursos para o financiamento da saúde, não respondem à pergunta motriz deste estudo, no sentido de que não abordam aspectos relativos às metodologias para alocação equitativa de recursos financeiros para o setor saúde. Entre estes últimos resultados excluídos, por um lado, grande parte trata sobre o modo como se dão os gastos com políticas públicas de saúde e os impactos de mecanismos distributivos no resultado dessas políticas, mas não se referem a como tais mecanismos foram elaborados pelos respectivos governos. Em outros casos, muitos dos estudos excluídos nessa etapa dizem respeito a como as reformas de sistemas de saúde impactam no funcionamento e na divisão de atribuições e responsabilidades dos diferentes níveis de atenção à saúde ou de governo.

Por fim, após a remoção dos artigos que estavam em duplicata, chegou-se ao número final de 19 publicações científicas que tangenciam o tema ou tratam diretamente sobre experiências voltadas a metodologias para alocação de recursos financeiros em saúde. Após a leitura na íntegra dos 19 artigos incluídos nesta revisão, extraíram-se deles os seguintes conteúdos: autor(es), título, objetivo(s) do estudo, método(s) utilizado(s) pelos autores na construção do estudo e a metodologia de alocação utilizada ou descrita pelos autores com suas dimensões (quando essas dimensões eram apresentadas no artigo) (quadro 2).

Quadro 2. Artigos incluídos na revisão: autores, objetivos, método dos estudos e como esses estudos abordam a alocação equitativa de recursos em saúde. Março, 2019

\begin{tabular}{|c|c|c|c|c|}
\hline $\begin{array}{l}\text { Base/ } \\
\text { Portal }\end{array}$ & Autor(es) & Objetivo(s) & Método do estudo & $\begin{array}{l}\text { Abordagem sobre a metodologia de } \\
\text { alocação de recursos em saúde }\end{array}$ \\
\hline \multirow[t]{3}{*}{ Scopus } & $\begin{array}{l}\text { Battesini } M \text {, } \\
\text { Andrade } C L T, \\
\text { Seta } \mathrm{MH}\end{array}$ & $\begin{array}{l}\text { Apresentar uma análise do financiamento federal } \\
\text { das ações de Vigilância Sanitária e do seu potencial } \\
\text { distributivo }\end{array}$ & $\begin{array}{l}\text { Estudo longitudinal } \\
\text { retrospectivo }\end{array}$ & Per capita \\
\hline & Vazquez DA & $\begin{array}{l}\text { Analisar os impactos dos mecanismos de regulação } \\
\text { federal - vinculação de receitas e transferências } \\
\text { condicionadas - sobre o financiamento em saúde }\end{array}$ & $\begin{array}{l}\text { Análise normativa } \\
\text { e dados retirados } \\
\text { do Siops }\end{array}$ & $\begin{array}{l}\text { Questões demográficas, sociossanitárias e } \\
\text { epidemiológicas }\end{array}$ \\
\hline & $\begin{array}{l}\text { Hughes D, } \\
\text { Leethongdee S, } \\
\text { Osiri S }\end{array}$ & $\begin{array}{l}\text { Descrever as mudanças no esquema de financia- } \\
\text { mento, realizadas por meio das Reformas de Cober- } \\
\text { tura Universal no sistema de saúde tailandês }\end{array}$ & $\begin{array}{l}\text { Entrevistas quali- } \\
\text { tativas }\end{array}$ & Critérios populacionais, etários e DRGs \\
\hline
\end{tabular}


Quadro 2. (cont.)

\begin{tabular}{|c|c|c|c|c|}
\hline $\begin{array}{l}\text { Base/ } \\
\text { Portal }\end{array}$ & Autor(es) & Objetivo(s) & Método do estudo & $\begin{array}{l}\text { Abordagem sobre a metodologia de } \\
\text { alocação de recursos em saúde }\end{array}$ \\
\hline \multirow[t]{7}{*}{ Scopus } & $\begin{array}{l}\text { Arredondo A, } \\
\text { Orozco E }\end{array}$ & $\begin{array}{l}\text { Identificar os efeitos da descentralização no finan- } \\
\text { ciamento e na governabilidade da saúde no México, } \\
\text { a partir da perspectiva de fornecedores e usuários }\end{array}$ & $\begin{array}{l}\text { Transversal e } \\
\text { entrevistas de } \\
\text { profundidade }\end{array}$ & Necessidade de cada região/município \\
\hline & $\begin{array}{l}\text { Häkkinen U, } \\
\text { Järvelin J }\end{array}$ & $\begin{array}{l}\text { Desenvolver uma fórmula para alocação de subsí- } \\
\text { dios do governo central a serviços de saúde munici- } \\
\text { pais e para cuidado com idosos na Finlândia }\end{array}$ & $\begin{array}{l}\text { Métodos estatís- } \\
\text { ticos e economé- } \\
\text { tricos. }\end{array}$ & Metodologia estatística e econométrica \\
\hline & Bossert TJ et al. & $\begin{array}{l}\text { Analisar a relação entre descentralização e equidade } \\
\text { na alocação de recursos na Colômbia e no Chile }\end{array}$ & $\begin{array}{l}\text { Abordagem } \\
\text { decision space }\end{array}$ & Per capita \\
\hline & Clavería A et al. & $\begin{array}{l}\text { Problematizar um modelo com uma nova alocação } \\
\text { de recursos, que facilite uma maior capacidade de } \\
\text { resolução na atenção primária e, assim, melhorar a } \\
\text { eficiência na área }\end{array}$ & Ensaio & $\begin{array}{l}\text { Per capita com base no gasto real por } \\
\text { pessoa, deve se ajustar à estrutura demo- } \\
\text { gráfica, dispersão geográfica, necessidades } \\
\text { de saúde. }\end{array}$ \\
\hline & Martínez G & $\begin{array}{l}\text { Descrever os mecanismos de alocação e compra do } \\
\text { Seguro Popular, a maneira como eles operam e os } \\
\text { controles que são dados sobre eles }\end{array}$ & Ensaio & $\begin{array}{l}\text { Diversos critérios como oportunidade e } \\
\text { qualidade, como distância ou tempo até } \\
\text { pontos de atendimento e certeza no aces- } \\
\text { so a medicamentos e consultas }\end{array}$ \\
\hline & $\begin{array}{l}\text { Vieira FS, } \\
\text { Zucchi P }\end{array}$ & $\begin{array}{l}\text { Analisar a aplicação direta de recursos financeiros } \\
\text { pelos entes federados para a aquisição de medica- } \\
\text { mentos no Sistema Único de Saúde }\end{array}$ & Quantitativo & Per capita \\
\hline & Qin W et al. & $\begin{array}{l}\text { Explorar como o Subsídio de Saúde do Governo } \\
\text { (Government Health Subsidy - GHS) foi alocado em } \\
\text { diferentes grupos socioeconômicos e o desempenho } \\
\text { do sistema de saúde em termos de alocação }\end{array}$ & Quantitativo & $\begin{array}{l}\text { Não foca na metodologia de alocação dos } \\
\text { recursos, apenas testa a metodologia para } \\
\text { constatar se está sendo equânime ou não }\end{array}$ \\
\hline \multirow[t]{3}{*}{ PubMed } & $\begin{array}{l}\text { Lima LD, } \\
\text { Andrade CLT }\end{array}$ & $\begin{array}{l}\text { Investigar as condições de financiamento do SUS } \\
\text { nos municípios com mais de } 100 \text { mil habitantes de } \\
\text { forma ampla }\end{array}$ & $\begin{array}{l}\text { Análise estatística } \\
\text { de dados coleta- } \\
\text { dos por meio do } \\
\text { SIOPS }\end{array}$ & $\begin{array}{l}\text { Indicadores sobre população, cobertura de } \\
\text { programas, oferta e produção de serviços, } \\
\text { entre outros }\end{array}$ \\
\hline & $\begin{array}{l}\text { Arredondo A } \\
\text { et al. }\end{array}$ & $\begin{array}{l}\text { Identificar as tendências e os efeitos da descentra- } \\
\text { lização nas políticas de financiamento da saúde no } \\
\text { México }\end{array}$ & $\begin{array}{l}\text { Entrevistas de pro- } \\
\text { fundidade e análise } \\
\text { documental }\end{array}$ & Necessidade de cada região/município \\
\hline & Smith PC & $\begin{array}{l}\text { Usar a experiência inglesa de alocação financeira } \\
\text { em saúde, com foco na alta complexidade, como } \\
\text { um estudo de caso }\end{array}$ & $\begin{array}{l}\text { Análise da legisla- } \\
\text { ção, das fórmulas } \\
\text { aplicadas e da } \\
\text { história da aplica- } \\
\text { ção dessas medi- } \\
\text { das para rateio dos } \\
\text { recursos }\end{array}$ & $\begin{array}{l}\text { Aponta soluções para resolver o problema } \\
\text { da iniquidade na distribuição de recursos, } \\
\text { a identificação das ações de cuidado em } \\
\text { saúde necessárias, além da identificação } \\
\text { dos grupos vulneráveis e sua localização } \\
\text { geográfica }\end{array}$ \\
\hline \multirow[t]{3}{*}{ Bireme } & $\begin{array}{l}\text { Smith N, } \\
\text { Church J }\end{array}$ & $\begin{array}{l}\text { Identificar fatores que facilitaram a adoção de finan- } \\
\text { ciamento com bases populacionais na província de } \\
\text { Alberta }\end{array}$ & $\begin{array}{l}\text { Pesquisa qualita- } \\
\text { tiva, com foco em } \\
\text { estudos de caso }\end{array}$ & $\begin{array}{l}\text { Características demográficas e neces- } \\
\text { sidades de saúde de cada localidade da } \\
\text { província }\end{array}$ \\
\hline & $\begin{array}{l}\text { Thomas S, } \\
\text { Okorafor OA, } \\
\text { Mbatsha S }\end{array}$ & $\begin{array}{l}\text { Analisar os progressos alcançados no financiamen- } \\
\text { to equitativo da Atenção Primária à Saúde (APS) } \\
\text { na África do Sul e avaliar as barreiras ao progresso } \\
\text { futuro }\end{array}$ & $\begin{array}{l}\text { Qualiquantitativo: } \\
\text { avaliação longitu- } \\
\text { dinal e transversal } \\
\text { do financiamento }\end{array}$ & Necessidades de saúde de cada região \\
\hline & $\begin{array}{l}\text { Javier Raña K, } \\
\text { Juan-Carlos } \\
\text { Ferrer O, Paula } \\
\text { Bedregal G }\end{array}$ & $\begin{array}{l}\text { Propor um modelo que aloca recursos para os Cen- } \\
\text { tros de Saúde das comunidades mais populosas na } \\
\text { região metropolitana de Santiago do Chile, sendo } \\
\text { baseado em uma forma equitativa, eficiente e trans- } \\
\text { parente }\end{array}$ & $\begin{array}{l}\text { Modelo Teórico } \\
\text { Quantitativo }\end{array}$ & $\begin{array}{l}\text { Prestações programadas - controles } \\
\text { médicos e outras consultas de rotina em } \\
\text { que os profissionais da saúde influenciam } \\
\text { a demanda; Morbidade - atenção de de- } \\
\text { manda espontânea, atenção de morbidade } \\
\text { e urgência, sendo originadas pelo paciente }\end{array}$ \\
\hline
\end{tabular}


Quadro 2. (cont.)

\begin{tabular}{|c|c|c|c|c|}
\hline $\begin{array}{l}\text { Base/ } \\
\text { Portal }\end{array}$ & Autor(es) & Objetivo(s) & Método do estudo & $\begin{array}{l}\text { Abordagem sobre a metodologia de } \\
\text { alocação de recursos em saúde }\end{array}$ \\
\hline \multirow[t]{3}{*}{ Bireme } & Zúñiga Fajuri A & $\begin{array}{l}\text { Propor um modelo de racionamento de recursos } \\
\text { de saúde que permita implementar as mudanças } \\
\text { necessárias para alcançar os objetivos de equidade } \\
\text { e eficiência que todo sistema de saúde procura } \\
\text { atualmente }\end{array}$ & $\begin{array}{l}\text { Econométrico } \\
(\mathrm{QALY})\end{array}$ & Idade e status econômico \\
\hline & $\begin{array}{l}\text { Arredondo A, } \\
\text { Parada I }\end{array}$ & $\begin{array}{l}\text { Identificar os efeitos da descentralização nas polí- } \\
\text { ticas de financiamento em três países da América } \\
\text { Latina: México, Nicarágua e Peru }\end{array}$ & $\begin{array}{l}\text { Quantiqualitativo: } \\
\text { dados secundá- } \\
\text { rios e entrevistas } \\
\text { diretas }\end{array}$ & $\begin{array}{l}\text { As diferentes categorias de análise para } \\
\text { cada país foram: famílias, empresas, go- } \\
\text { verno federal, governo estadual, impostos } \\
\text { governamentais, créditos governamentais, } \\
\text { doação do governo e outras doações }\end{array}$ \\
\hline & Neves MCP & $\begin{array}{l}\text { Discutir a problemática da 'alocação dos recursos } \\
\text { em saúde' como uma das questões mais urgentes } \\
\text { desenvolvidas no âmbito da bioética }\end{array}$ & Ensaio & $\begin{array}{l}\text { Dignidade humana, participação, equidade } \\
\text { e solidariedade }\end{array}$ \\
\hline
\end{tabular}

\section{Discussão}

Segundo os resultados desta revisão, é possível perceber que há uma variada forma de apropriação, ainda que seja em poucos estudos, do tema das metodologias de alocação equitativa de recursos em saúde. Entre os estudos, é possível perceber que a discussão transita de uma compreensão imprecisa sobre a ideia de 'alocar' até os estudos que focam diretamente na apreensão bem delimitada da alocação de forma equitativa. Do ponto de vista dos objetivos dos estudos revisados, podem-se identificar sete blocos de intencionalidades dos pesquisadores ao abordar a temática.

O primeiro bloco de estudos é aquele que considera, apenas tangencialmente, o tema em sua análise. Assim, neste bloco, com apenas três estudos ${ }^{10-12}$, a discussão da alocação se confunde com o 'financiamento de sistemas locorregionais e a distribuição dos recursos entre os entes federal-subnacionais'. $\mathrm{O}$ segundo bloco de estudos recorta a compreensão da alocação a partir da perspectiva da tríade 'financiamento-regulação-transferên-

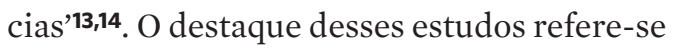
aos resultados das regras (critérios e condicionantes) que interferem na distribuição, apropriação e no uso de recursos vinculados à saúde nos orçamentos municipais. No terceiro bloco, com apenas um estudo representativo dessa temática, está aquele que, ainda restrito à delimitação pouco clara entre alocação e financiamento, delineia as 'Mudanças nos esquemas de financiamento'15. Para estes autores, a alocação está circunscrita à lógica das reformas de financiamento dos sistemas de saúde quando visam a descrever as mudanças no esquema de financiamento. Por fim, ainda naqueles estudos que mesclam de forma imprecisa financiar-alocar, há um quarto bloco que é aquele que situa a discussão da alocação de recursos intrínseca à organização entre 'financiamento-descentralização'16-19.

Nesses quatro primeiros blocos, percebe-se que a ideia de alocar ainda não está bem clara, ou talvez especificada. É pertinente atentar para a diferença entre 'financiar' e 'alocar'. Financiar, especialmente em sistemas de saúde nacionais ${ }^{20}$, está relacionado com acúmulo de recursos (seja um fundo, uma reserva ou assemelhados), enquanto alocar está relacionado com a destinação de recursos já existentes, ou seja, após a coleta desses recursos para onde eles serão distribuídos ${ }^{4}$. Por esses conceitos terem esse caráter relacional, e, portanto, relativo, por 
vezes, confundem-se no senso comum e são refletidos nos artigos que, em certa medida, adotam essa 'semelhança' entre o termo alocação e financiamento, dificultando sua análise.

No debate sobre o processo alocativo, os 'critérios da alocação' são o tema mais delicado no que se refere ao alcance da equidade. Contudo, a leitura regulacionista da alocação tem como discurso latente a 'eficiência' em termos de garantir aos produtores e consumidores suas 'exigências', ou seja, aos primeiros, maximizando lucro, e aos outros, maximizando satisfação (utilidade). Assim, qual seria o local da equidade? O que parece estar subjacente ao debate alocativo sobre essa 'chave' é a naturalização da ideia de agentes 'racionais' e de 'comportamento maximizador' entre os entes subnacionais como se estes tivessem acesso à informação completa e uma postura imparcial nessa dinâmica competitiva ${ }^{21}$. Ora, pensar a alocação sob esses pressupostos pode obscurecer o papel da intencionalidade desses 'agentes', especialmente sobre a priorização da equidade em detrimento da eficiência.

Do ponto de vista da descentralização, é importante ser pontuado que o processo alocativo é essencial para esse preceito e que, no Brasil, resulta da discussão sobre a solidariedade entre os entes federados e o financiamento tripartite. Contudo, Nunes et al. ${ }^{22}$, desde 2001, já apontava que um dos grandes problemas para o desenvolvimento de metodologias alocativas de recursos no Brasil tem sido a falta de sistemas de informações integrados, especialmente quando se trata de entes federativos distintos, tornando a análise das desigualdades em saúde um grande desafio. É por isso que o problema alocação-descentralização ainda persiste, sendo necessário, no caso brasileiro, criar novas formas de captação de informação regular e automática, com sistemas de informação adequadamente alimentados para conferir as informações ideais ao refinamento do conceito de equidade.

Ao adentrar nos estudos que já delimitam bem o conceito de alocação, em detrimento do conceito de financiamento, outros três blocos compõem melhor o tema e dialogam mais diretamente com a pergunta da revisão. Assim, o quinto bloco de estudos é aquele que apresenta 'proposições e/ou desenvolvimento de fórmulas' ${ }^{23-26}$ para alocação de recursos em saúde em cenários de diferentes sistemas de saúde. Um sexto bloco de estudo se aproxima mais da temática, à medida que foca diretamente na 'testagem da aplicação (alocação) dos recursos' ${ }^{\mathbf{2 7}, 28}$. E, por fim, o sétimo e último bloco atinge o cerne da discussão encetada, focando na 'alocação por equidade'29-31. Assim, três estudos se centram nessa questão. O primeiro explora como o Subsídio de Saúde do Governo (Government Health Subsidy - GHS) foi alocado em diferentes grupos socioeconômicos e o desempenho do sistema de saúde em termos de alocação desse subsídio para diferentes tipos de serviços de saúde. O segundo analisa os progressos alcançados no financiamento equitativo da Atenção Primária à Saúde (APS) na África do Sul e avalia as barreiras ao progresso futuro. O terceiro discute a problemática da 'alocação dos recursos em saúde' como uma das questões mais urgentes desenvolvidas no âmbito da bioética. Considera que a questão deve ser analisada em termos de justiça social e de responsabilização moral, tomando-se como ponto de partida a corroboração do direito à saúde.

Ao enfatizar as metodologias, é esperado que proposições e/ou desenvolvimento de fórmulas, suas testagens, simulações ou demonstrações de aplicação dos recursos possam evidenciar se discernem ou não equitativamente os processos de transferência. Contudo, salvo raras exceções, considera-se uma discussão, ainda que abstrata (contudo necessária), sobre como operacionalizar os métodos de alocação equitativa e as simulações com diferentes critérios justamente para serem evitadas diversas formas de aumento das desigualdades, como a drenagem de recursos públicos para subsidiar segurados de planos e de seguros privados ${ }^{32}$, por exemplo. Em que pese a necessidade de que as metodologias devem levar em consideração a 
tríade proposição-testagem-aplicação para provarem a equidade de suas competências alocativas ${ }^{33}$, este debate tende a ser 'tenso' na medida em que, em cenários onde não há incremento orçamentário, diversos argumentos que desconstroem os fundamentos éticos da construção da equidade (como a solidariedade) são levantados, aventando, por exemplo, que os vazios assistenciais, as gestões com dificuldades técnicas ou ineficientes (em termos regulacionistas) não mereceriam ganhar mais recursos.

Do ponto de vista do método utilizado pelos autores para tratar da temática, uma miscelânea foi utilizada, desde abordagens menos rigorosas, ou também chamadas descritivo-reflexivas sobre o tema, até aqueles métodos clássicos das ciências estatísticas ou econométricas, ou, ainda, no bojo das abordagens qualitativas.

No primeiro grupo dos estudos, que apresenta um caráter descritivo-reflexivo, predominou o 'ensaio' como modalidade textual utilizada ${ }^{11,23,31}$. Partindo-se para um segundo grupo de estudos, com metodologias mais rigorosas, quatro deles optaram por uma 'abordagem quantitativa-descritiva' da alocação de

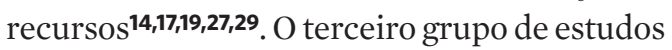
foi aquele que optou pelo uso de 'abordagens quantitativas clássicas utilizadas nas ciências econômicas ou na econometria'. Assim, três estudos se assentam nesses métodos ${ }^{24-26}$. O quarto grupo refere-se àqueles estudos que metodologicamente inovam, a exemplo dos que usam 'abordagens qualitativas' $15,16,18$, pouco utilizadas no campo das ciências econômicas. O quinto e último grupo de propostas metodológicas traz 'abordagens híbridas entre métodos quantitativos e qualitativos'10,12,17.

É importante perceber que, entre as escolhas metodológicas dos artigos revisados, esse amplo espectro metodológico traz mais possibilidades de transladar a equidade do ponto conceitual para o operacional. Esse resultado parece ser importante para construir uma narrativa de levar a equidade desde os seus elementos mais abstratos até os mais concretos com métodos que se adequam às propostas de captura da equidade nesses diferentes aspectos. Espera-se que novos estudos, inclusive, tentem arrojar nas análises empreendidas que mesclem as abordagens, conforme realizado pelo quinto grupo, na tentativa de não restringir o debate apenas às ciências estatísticas ou à mera matematização apenas ${ }^{34}$.

Ao focar nas metodologias de alocação de recursos e suas dimensões e/ou indicadores, os estudos também apresentam grande diversidade, desde aqueles que não explicitam o método (do ponto de vistas de cálculo, dimensões ou indicadores) até aqueles que detalham todas as características do método com foco no(s) aspecto(s) que cada um privilegia, do ponto de vista operacional, para garantir a equidade. Esses estudos podem ser organizados em 8 grupos por nível de aproximação e refinamento do debate sobre a metodologia.

O primeiro grupo se reserva aos estudos que 'não descrevem a metodologia, mas a testam'. É o caso do estudo do Qin et al. ${ }^{29}$, que se dedica a testar a garantia da equidade na metodologia utilizada para alocação de recursos no sistema de saúde chinês.

O segundo grupo, na tentativa teórica de problematizar o tema, discute as 'implicações (bio)éticas da alocação', especialmente no cuidado em operacionalizar o princípio da equidade ${ }^{31}$.

Em seguida, o terceiro grupo, certamente o mais frequente entre os estudos que se dedicam à temática, é composto por aqueles que 'focam o componente per capita sem avançar na discussão da equidade na alocação'10,19,23,27. Nesses estudos, o enfoque metodológico ressalta o critério per capita, especialmente para o fomento da descentralização das ações ${ }^{10}$, mas reconhece que o enfoque nesse indicador não garante nem amplia a equidade da alocação dos recursos, não sendo, portanto, o suficiente para tal operacionalização' ${ }^{19}$, especialmente na aquisição de medicamentos via SUS27. Reconhecem que o per capita com base no gasto real por pessoa deve se ajustar a diversas dimensões essenciais à alocação, como: estrutura demográfica, dispersão geográfica, necessidades de 
saúde (morbidade, ajuste para o último ano de vida, nível educacional, privação etc.), e, ainda, ao catálogo de serviços prestados e seu grau de complexidade, estrutura instalada, entre outros $^{23}$. Trata-se de, portanto, reconhecer que o per capita é o início de uma metodologia, mas nunca deve ser seu fim.

O quarto grupo de estudos apresenta a 'orçamentação incremental' como uma possibilidade. Ainda retoma, especialmente em tempos de crise, o incremento de recursos (novos investimentos) como essencial em quaisquer setores, especialmente na saúde, onde os custos são crescentes. Neste estudo, a orçamentação incremental na atenção básica passou a disputar recursos orçamentários com outras áreas, o que, sem uma análise das desiguais necessidades de saúde de cada região, pode promover problemas do ponto de vista do peso de um setor no orçamento geral ${ }^{30}$.

Durante muito tempo, os estudos brasileiros, na abordagem da alocação de recursos no SUS, restringiam-se aos cálculos tradicionais (per capita) e à demonstração da diminuição da desigualdade por meio da orçamentação incremental ${ }^{35}$. Mesmo considerando essa questão importante (e necessária), faz-se mister o avanço do debate metodológico para além desses marcos, já considerados 'iniciais' atualmente. Ugá e Lima 4 já apontavam que os métodos tradicionais de alocação de recursos têm sido substituídos por sistemas mistos ou complementados. Contudo, o que vem sendo continuamente demonstrado é que, na maioria das vezes, quando se avança na discussão metodológica, ela tem recaído na adição de propostas de indicadores/dimensões que aumentam as desigualdades, como no caso das alocações que têm por critério de pagamento o desempenho (pay for performance), a captação, a produção, entre outros mecanismos iníquos.

À medida que os estudos vão rebuscando suas dimensões para reelaborar as metodologias, a pauta da equidade e sua operacionalidade vão se delineando no método. O quinto grupo de estudo é aquele em que se enfocam as 'questões sociodemográficas, sociossanitárias e epidemiológicas' como principais dimensões para elencar os indicadores no método para garantir equidade (ainda que de forma inicial) $^{\mathbf{1 3}, 15,26}$. Vazquez ${ }^{\mathbf{1 3}}$ destaca a insuficiência dos critérios de repasse do Piso da Atenção Básica para garantir equidade, por desconsiderarem justamente essas dimensões. O uso de critérios como a idade ou o status econômico do paciente, ao definir as regras de acesso para garantir ${ }^{\mathbf{2 6}}$ a equidade, também é apresentado como uma alternativa. Outros autores ${ }^{15}$, ao estudarem o sistema de saúde tailandês, defendem os critérios populacionais, etários, além de DRGs (Diagnosis Related Groups), para alta e média complexidade e atenção hospitalar. Quando se pensa em alocar recursos, especialmente em saúde, é essencial abandonar o caráter regressivo das formas de tributação para alcançar progressividade, já que, em termos de operacionalização da equidade, a alocação teria mais potência equitativa.

O ponto de inflexão da narrativa nos estudos acontece no sexto grupo de estudos quando é problematizada nas metodologias a dimensão da 'necessidade de saúde como centro da discussão' do alcance de uma alocação equitativa. De forma mais modesta ou em discussões mais refinadas, o debate sobre as necessidades de saúde por cada região, para a maior parte dos estudos, é central, e sua operacionalização um desafio. Os estudos mais modestos se reservam a identificar nos sistemas de informação de seus governos e sistemas de saúde a possibilidade de indicadores que delineiem (nem que seja minimamente) o que seria a 'necessidade de saúde', como, por exemplo, os seguintes indicadores: população, cobertura de programas, oferta e produção de serviços ${ }^{12,14}$. Outros estudos ${ }^{16-18}$, de forma intermediária, avançam na discussão através da implantação de mecanismos de ajuste anual de acordo com a necessidade de cada região/município ${ }^{17}$. Outros estudos, mais sofisticados, revisitam o histórico das fórmulas de alocação de recursos ou revisitam as fórmulas na tentativa de melhorar o sistema de alocação na busca da equidade. Esses são os exemplos do estudo 
sobre o sistema finlandês ${ }^{25}$, que dividiu os tipos de serviços de saúde em 6 grupos, desenvolvendo indicadores de necessidades de saúde para cada um deles, seja por meio de estudos econométricos, seja por características demográficas e do estudo chileno, ainda que de forma muito microeconômica ${ }^{24}$. Neste, dois tipos de atividades eram consideradas dimensões com indicadores sobre como operar equitativamente: 'prestações programadas' controles médicos e outras consultas de rotina em que os professionais da saúde influenciam a demanda; 'morbidade' - atenção de demanda espontânea, atenção de morbidade e urgência, sendo originadas pelo paciente. Essas duas atividades podem ser desagregadas em elementos que determinam os custos e a demanda.

O sétimo grupo de estudo parte para uma composição mais elaborada da equidade na alocação dos recursos que tenta 'mediar o macro como microeconômico'. Representado por um estudo $^{\mathbf{2 3}}$, o autor sugere diversas dimensões de análise que transitam entre a alocação realizada por critérios grupais até os individuais. Assim, critérios de gerenciamento geral, dos gestores em nível central e local, até a gestão da clínica realizada pelo profissional, devem ser indicadores a serem levados em consideração. Ademais, o grau de satisfação dos usuários, a capacidade de resolução dos problemas de ordem clínica, a qualidade da atenção e a eficiência da assistência são indicadores locais importantes segundo esse estudo. Isso tudo não estaria desvinculado da necessidade de uma avaliação externa e, segundo o autor, de um sistema de remuneração.

O oitavo e último grupo de estudos é aquele no qual a 'operacionalização da equidade está mais desenvolvida metodologicamente ${ }^{\prime 11,16,28}$. Assim, os autores apontam que o melhoramento e o alcance da equidade na alocação são processos históricos e requerem esforços contínuos, em todos os governos, em adensar a metodologia garantindo a incorporação de novos indicadores (e até criando novos processos de coleta de informação regular e contínua) para refinar a equidade operacionalmente.
Para alguns autores"1, isso não está desvinculado de novos investimentos, logo, projetar modelos sobre o custo incremental é essencial, para daí partir para quais critérios sobre $o$ atendimento das populações remotas ou marginalizadas devem ser utilizados. Ainda, a definição deve incluir elementos de 'oportunidade' e 'qualidade', como 'distância' ou 'tempo até pontos de atendimento' e 'certeza no acesso a medicamentos e consultas'. Além disso, para garantir a lógica de 'sistema', é fundamental adotar procedimentos que vinculem os cuidados primários aos hospitais, o baixo custo para o médico e o paciente. Ademais são necessários mecanismos interativos de informação e comunicação entre hospitais e jurisdições de saúde e outras agências estaduais em um sistema interativo de vinculação a dados da previdência social e outros programas, bem como a provedores privados para o refino do processo alocativo.

Outra experiência bem desenvolvida se refere às dimensões utilizadas no estudo comparativo entre os sistemas de saúde no México, na Nicarágua e no Peru' ${ }^{16}$. Os autores identificam que, em países latino-americanos, as dimensões fundamentais para a metodologia ser equitativa são 6: 'famílias', através dos aportes monetários diretamente da renda familiar; 'empresas', através dos aportes monetários diretamente dos empregadores (em salários diretos e indiretos etc.); 'impostos governamentais', através das amortizações monetárias de impostos federais e locais; 'créditos governamentais', por meio dos aportes monetários de créditos com bancos internacionais via governo federal; 'doações do governo', por meio das contribuições monetárias de doações de agências internacionais via federal ou governo local; e 'doações de outras entidades', como no caso dos aportes monetários diretamente de doações de $\mathrm{ONG}$ internacionais e nacionais.

Certamente, o estudo mais completo e que melhor delineia uma metodologia de alocação equitativa é o estudo sobre o sistema de saúde inglês' ${ }^{\mathbf{2 8}}$, que detalha historicamente as fases 
do aperfeiçoamento por mais de 40 anos do Resource Allocation Working Party (RAWP). Em seus primeiros anos, a metodologia tinha como indicadores o tamanho da população com ajuste para características demográficas (idade e sexo específicos para cada especialidade); uma ponderação adicional para necessidade clínica adicional (medida pelas taxas de mortalidade locais padronizadas para as especialidades específicas), com um ajuste para variações nos preços de insumos dos serviços locais. No seu desenvolvimento, foi incorporada a taxa padronizada de doenças limitantes de longa duração (menos de 75 anos), a taxa de mortalidade padronizada (menos de 75 anos), a proporção de desempregados economicamente ativos, a proporção de idade do aposentado morando sozinho e a proporção de dependentes em famílias com um único cuidador. Em uma terceira fase, incorporaram-se medidas de qualidade dos serviços de saúde, acesso a serviços de saúde e fatores fora do controle direto do sistema de saúde (riqueza, estilo de vida, considerações genéticas e ambientais). Em um quarto momento de aperfeiçoamento, incorporou-se o indicador de 'mortalidade evitável', definido como o número de anos de vida perdidos com menos de 75 anos durante um período de três anos. Nos períodos mais recentes, a alocação de recursos diretamente para os médicos generalistas passou a ser o debate, direcionando-se para aqueles médicos que optaram por se tornar 'administradores de fundos'. Após essa medida, adicionou-se o 'risco orçamentário' como indicador, definido como a propensão para as despesas reais variarem das despesas previstas. Então, desde 2008, a 'alocação de recursos baseada em pessoas' tem sido a discussão, e sua essência é desenvolver pagamentos de captação individual por idade e sexo, e ajustá-los a qualquer medida inequívoca e universalmente registrada de desvantagem (como recebimento de bem-estar) e a qualquer indicador verificável de diagnóstico prévio de doença.

Ainda que essa metodologia seja a mais aprimorada, os estudos relativos ao RAWP e sua adaptabilidade a outros contextos 7,8 já apontavam limitações importantes como, por exemplo, a característica das desigualdades no Brasil. Além disso, é prudente salientar que o processo de 'refino' da equidade apresentado no desenvolvimento dessa metodologia tem mais ligação com a incorporação de elementos de mercado no sistema inglês ${ }^{36}$ do que, necessariamente, com a preocupação de delimitar melhor a vulnerabilidade dos grupos sociais, focando-se nos indivíduos. Recentemente, no Brasil, o modelo de alocação (insistentemente chamado de 'financiamento') para a atenção primária, por exemplo, mimetiza várias características do RAWP, entre elas, o problema da centralidade do aspecto operacional do sistema - o cadastramento -, e não a equidade. Isso porque os autores ${ }^{\mathbf{3 7}}$ dessa proposta enfatizam a problemática da população cadastrada do SUS, enquanto pouco esforço é destinado aos problemas de equidade que a forma de alocação histórica do SUS deixou de considerar.

Mesmo sob a variabilidade de achados que este estudo apresenta, é fundamental reconhecer as limitações que a captura e a análise do objeto apresentam. Em primeiro lugar, o uso de uma revisão do tipo integrativa, apesar de demonstrar um panorama aproximado sobre como um objeto vem sendo estudado, apresenta como limite não focar essencialmente no objeto per si. Isso demonstra o quanto alguns artigos incluídos na revisão não apresentam diretamente as metodologias, mas apenas 'circundam' o objeto, trazendo elementos subsidiários para pensar a alocação equitativa, mas sem demonstrar um método acabado e suas dimensões/indicadores. Isso implica dizer que, em estudos posteriores, ampliar o escopo (através de outras bases de dados) e usar outros métodos de revisão de maior precisão na apreensão (revisões sistemáticas, por exemplo) podem ser o caminho para avançar na discussão.

Outro aspecto que chamou atenção na organização da estratégia de busca dos estudos foi a indexação indevida de certos descritores feita pelos autores dos estudos. Isso dificultou 
a recuperação de estudos feitos por autores consagrados na economia da saúde. Essa é uma limitação importante do processo metodológico, que, porventura, deixou de fora textos considerados clássicos da discussão sobre o tema. Contudo, mesmo sob essa ressalva, procedeu-se à incorporação desse acúmulo do debate no âmbito brasileiro por meio do resgate de seus principais pesquisadores no diálogo com os artigos incluídos. Além disso, é pertinente ressaltar que, devido às estratégias de busca utilizadas, foi possível recuperar estudos que, certamente, não faziam parte do debate corrente na literatura brasileira, trazendo as experiências internacionais e oxigenando a discussão.

Neste debate não se deve furtar da contribuição de outros estudos de revisão sistematizada na literatura mundial sobre a alocação em saúde, mas identificar seus objetos e, também, suas limitações. Alguns estudos versam sobre diversos aspectos: sobre definições de equidade nos cuidados em saúde e na classificação dos domínios que a equidade apresenta ${ }^{38}$; sobre os critérios para alocação de recursos (sem mencionar o método) ${ }^{\mathbf{3 9}, \mathbf{4 0}}$; sobre critérios dispostos nas legislações que cada país ${ }^{41}$ utiliza para alocar; sobre os fatores que influenciam nas decisões de alocação por gerentes ${ }^{\mathbf{4 2}}$; sobre os estudos de avaliação econômica ${ }^{43-45}$; sobre atributos de saúde e não relacionados à saúde para priorizar a tomada de decisão ${ }^{46}$; sobre os estudos que criaram uma metodologia única, segundo os dados de cada país ${ }^{47}$; e aqueles que analisam os desafios éticos enfrentados pelos gestores do SUS ${ }^{48}$.

Ainda que se reconheça o valor desses estudos em circundarem o tema, é necessário relembrar que eles não entraram nesta revisão, pois o foco não foi revisar revisões já realizadas. Mesmo que os estudos anteriormente citados fossem estudos primários, eles não focam no método de alocação, garantindo, assim, a originalidade deste estudo aqui realizado, que reside, especificamente, em ter como objeto a metodologia da alocação (desde sua concepção à aplicação).
Mesmo assim, é notório reconhecer que a estratégia metodológica utilizada para este objeto (metodologias de alocação equitativa de recursos financeiros em saúde) é um tema de pesquisa desafiante, e, justamente por isso, poucos estudos na literatura disponibilizada nessas três bases/portais revisadas foram encontrados. Isso revela a importância da adaptação do melhor tipo de revisão em função do objeto pesquisado e sua apresentação na literatura científica. Assim, entende-se que, mesmo sob as limitações do método empreendido, este ainda é o melhor para capturar esse objeto nesse estágio de desenvolvimento da pesquisa científica.

Após ponderadas as limitações deste estudo, é fundamental apontar que suas possibilidades são incontestes ao debate da alocação, especialmente no Brasil, em tempos de crise econômica de longa duração. Por mais que já esteja bastante ratificado na literatura científica que o problema central da questão macroeconômica em saúde seja o desfinanciamento do seu setor público ${ }^{49}$, alocar recursos admite uma saída, ainda que paliativa. Assim, alocar equitativamente significa, além de melhorar a distribuição dos parcos recursos destinados ao sistema público de saúde é, em última instância, fazer valer a lei e sua consonância constitucional idealizada e arduamente defendida pela reforma sanitária brasileira, desde o primeiro estudo sobre alocação no SUS, que discutiu o artigo 35 da Lei ${ }^{\circ}$ 8.080, realizado por Vianna e Piola ${ }^{50}$.

\section{Conclusões}

Pode-se dizer que as metodologias de alocação equitativas de recursos em saúde e suas dimensões são um objeto de estudo em delimitação e que as pesquisas na área ainda precisam avançar em propostas que considerem o que os achados desta revisão proporcionam, ou seja, considerar as implicações (bio)éticas da alocação de recursos, definindo claramente o conceito de equidade utilizado 
e operacionalizando coerentemente, tanto do ponto de vista da solidariedade como da participação.

Além desse pressuposto, as metodologias devem partir de um componente per capita, mas devem considerar a equidade, principalmente (mas não exclusivamente) através de um conjunto de fatores como a orçamentação incremental, as questões sociodemográficas, sociossanitárias e epidemiológicas, a captura das 'necessidade de saúde' (com uma definição clara e operacionalidade coerente) como centro da sua formulação, a mediação entre o macro como microeconômico e, sem dúvida, o entendimento de que esse é um processo que precisa estar sempre em desenvolvimento/ aperfeiçoamento.

Talvez esta última característica das metodologias seja a mais sensível, principalmente em cenários de descontinuidades político-administrativas de governos e em países latino-americanos de inserção dependente.
Certamente, se a metodologia não é constantemente melhorada ao longo dos anos de implementação, provavelmente, ela deixará de acompanhar a dinâmica da sociedade e, possivelmente, a equidade não alcançará o máximo de sua expressão, perpetuando as desigualdades na alocação e, sobretudo, comprometendo a efetividade do direito à saúde.

\section{Agradecimentos}

A Bruno Lopes Zanetta, por ter coletado parte dos dados relacionados à revisão.

\section{Colaboradores}

Carnut L (0000-0001-6415-6977)*, Mendes A (0000-0002-5632-4333)*, Leite MG (00000001-9305-9703)* contribuíram igualmente para a elaboração do manuscrito.

\section{Referências}

1. Brasil. Constituição da República Federativa do Brasil, de 5 de outubro de 1988. [acesso em 2018 nov 27]. Disponível em: http://www.planalto.gov.br/ccivil_03/ constituicao/constituicao compilado.htm.

2. Mendes A. A longa batalha pelo financiamento do SUS. Saúde Soc. 2013; 22(4):987-993.

3. Ocké Reis CO, Ribeiro JAC, Piola SF. Financiamento das Políticas Sociais nos anos 1990: O Caso do Ministério da Saúde. Brasília, DF: IPEA, 2001. (Texto para discussão N. 802).

4. Ugá MAD, Lima SML. Sistemas de alocação de recursos a prestadores de serviços a saúde. In: Fundação Oswaldo Cruz. A saúde no Brasil em 2030 - prospecção estratégica do sistema de saúde brasileiro: estrutura do financiamento e do gasto setorial. Rio de Janeiro: Fiocruz; Ipea; Ministério da Saúde; Secretaria de Assuntos Estratégicos da Presidência da República, 2013. p. 135-168, 2013. (v. 4).
${ }^{*}$ Orcid (Open Researcher and Contributor ID). 
5. Brasil. Emenda Constitucional ${ }^{\circ} 93$, de 8 de setembro de 2016. Altera o Ato das Disposições Constitucionais Transitórias para prorrogar a desvinculação de receitas da União e estabelecer a desvinculação de receitas dos Estados, Distrito Federal e Municípios. 2016. Diário Oficial da União. 9 Set 2016. [acesso em 2020 ago 24]. Disponível em: https://www2.camara. leg.br/legin/fed/emecon/2016/emendaconstitucional-93-8-setembro-2016-783591-norma-pl.html.

6. Brasil. Lei Complementar $n^{\circ} 141$, de 13 de janeiro de 2012. Regulamenta o $₫ 30$ do art. 198 da Constituição Federal para dispor sobre os valores mínimos a serem aplicados anualmente pela União, Estados, Distrito Federal e Municípios em ações e serviços públicos de saúde; estabelece os critérios de rateio dos recursos de transferências para a saúde e as normas de fiscalização, avaliação e controle das despesas com saúde nas 3 (três) esferas de governo; revoga dispositivos das Leis nos 8.080, de 19 de setembro de 1990, e 8.689, de 27 de julho de 1993; e dá outras providências. 2012, Diário Oficial da União. 14 Jan 2012. [acesso em 2020 ago 24]. Disponível em: http://www.planalto.gov.br/ccivil_03/leis/lcp/lcp141.htm.

7. Porto S, Martins M, Travassos C, et al. Avaliação de uma metodologia de alocação de recursos financeiros no setor saúde para aplicação no Brasil. Cad. Saúde Pública. 2007; 23(6):1393-1404.

8. Mendes A, Leite MG, Marques RM. Discutindo uma metodologia para a alocação equitativa de recursos federais para o Sistema Único de Saúde. Saúde e Soc. 2011; 20(3):673-690.

9. Soares CB, Hoga LAK, Peduzzi M, et al. Revisão integrativa: conceitos e métodos utilizados na enfermagem. Rev. Esc. Enferm. USP. 2014; 48(2):335-45.

10. Battesini M, Andrade CLT, Seta MH. Financiamento federal da vigilância sanitária no Brasil de 2005 a 2012: Análise da distribuição dos recursos. Ciênc. Saúde Colet. 2017; 22(10):3295-3306.

11. Martínez G. Política de asignación de recursos del Seguro Popular: análisis y recomendaciones. Salud pública México. 2016; 58(5):577-583.
12. Smith N, Church J. Shifting the lens: the introduction of population-based funding in Alberta. Healthc Manage Forum. 2008; 21(2):36-42.

13. Vazquez DA. Efeitos da regulação federal sobre o financiamento da saúde. Cad. Saúde Pública. 2011; 27(6):1201-1212.

14. Lima LD, Andrade CLT. Condições de financiamento em saúde nos grandes municípios do Brasil. Cad. Saúde Pública. 2009; 25(10):2237-48.

15. Hughes D, Leethongdee S, Osiri S. Using economic levers to change behaviour: The case of Thailand's universal coverage health care reforms. Social Science and Medicine. 2010; 70(3):447-454.

16. Arredondo A, Parada I. Health financing changes in the context of health care decentralization: the case of three Latin American countries. Rev. Saúde Pública. 2000; 34(5):449-460.

17. Arredondo A, Orozco E. Effects of health decentralization, financing and governance in Mexico. Rev. Saúde Pública. 2006; 40(1):152-160.

18. Arredondo A, Parada I, Orozco E, et al. Efectos de la descentralización en el financiamiento de la salud en México. Rev. Saúde Pública. 2004; 38(1):121-129.

19. Bossert TJ, Larrañaga O, Giedion U, et al. Decentralization and equity of resource allocation: Evidence from Colombia and Chile. Bull World Health Org. 2003; 81(2):95-100.

20. Conill EM. Sistemas comparados de saúde. In: Campos GWS et al. Tratado de saúde coletiva. São Paulo: Hucitec; Rio de Janeiro: Fiocruz, 2006, p. 563-613.

21. Meirelles DS. Teorias de mercado e regulação: por que os mercados e o governo falham?. Cadernos EBAPE. BR. 2010; 8(4):644-660.

22. Nunes A, Santos JRS, Barata RB, et al. Medindo as desigualdades em saúde no Brasil: uma proposta de monitoramento. Brasília, DF: OPAS. Ipea, 2001. 
23. Clavería A, Ripoli MA, López-Rodrigues A, et al. La cartera de servicios en atención primaria: un rey sin camisa. Informe SESPAS 2012. Gaceta sanitaria. 2012; 26(supl1):142-150.

24. Javier Raña K, Juan-Carlos Ferrer O, Bedregal Paula G. Modelo de asignación de recursos en atención primaria. Rev. méd. Chile. 2007; 135(1):54-62.

25. Häkkinen U, Järvelin J. Developing the formula for state subsidies for health care in Finland. Scand. J. Public Health. 2004; 32(1):30-39.

26. Zúñiga Fajuri A. Un modelo de adjudicación de recursos sanitarios para Chile. Acta bioethica. 2012; 18(2): 221-230.

27. Vieira FS, Zucchi P. Aplicações diretas para aquisição de medicamentos no Sistema Único de Saúde. Rev. Saúde Pública. 2011; 45(5):906-913.

28. Smith PC. Resource allocation and purchasing in the health sector: the English experience. Bull World Health Organ. 2008; 86(11):884-8.

29. Qin W, Xu L, Li J, et al. Estimating benefit equity of government health subsidy in healthcare Services in Shandong Province, China: a cross-sectional study. Int. J. Equity Health. 2018; 17(61):1-9.

30. Thomas S, Okorafor AO, Mbatsha S. Barriers to the equitable funding of primary healthcare in South Africa. Appl Health Econ Hea. 2005; 4(3):183-90.

31. Neves MCP. Alocação de recursos em saúde: considerações éticas. Bioética. 1999; 7(2):1-5.

32. Piola SF. Transferências de recursos federais do sistema único de saúde para estados, distrito federal e municípios: os desafios para a implementação dos critérios da Lei Complementar $n^{\circ}$ 141/2012. (Texto para discussão) Instituto de Pesquisa Econômica Aplicada. Brasília, DF; Rio de Janeiro: Ipea, [internet]. 2017. [acesso em 2019 nov 1]. Disponível em: http://repositorio.ipea.gov.br/bitstream/11058/7777/1/td_2298. pdf.
33. Pelegrini MLM, Castro JD, Drachler ML. Eqüidade na alocação de recursos para a saúde: a experiência do estado do Rio Grande do Sul, Brasil. Ciênc. Saúde Colet. 2005; 10(2):275-286.

34. Carnut L, Pires JSM, Mendes, A. 'Economia da Saúde' ou 'Economia Política da Saúde'? Em defesa de uma abordagem crítica marxista. [acesso em 2019 out 16]. Disponível em: https://sep.org.br/anais/2019/Sessoes-Ordinarias/Sessaol.Mesas1_10/Mesa9/093.pdf.

35. Souza RR. Redução das desigualdades regionais na alocação dos recursos federais para a saúde. Ciênc. Saúde Colet. 2003, 8(2):449-460.

36. Filippon J, Giovanella L, Konder M, et al. A “liberalização” do Serviço Nacional de Saúde da Inglaterra: trajetória e riscos para o direito à saúde. Cad. Saúde Pública. 2016 [acesso em 2019 nov 1]; 32(8):e00034716. Disponível em: https://www.scielo.br/pdf/csp/ v32n8/1678-4464-csp-32-08-e00034716.pdf.

37. Harzheim E, D’Avila OP, Ribeiro DC, et al. Novo financiamento para uma nova atenção primária à saúde no Brasil. Ciênc. Saúde Colet. 2020. 25(4):1361-1374.

38. Lane H, Sarkies M, Martin J, et al. Equity in healthcare resource allocation decision making: A systematic Review. Soc. Sci. Med., 2017; 175:11-27.

39. Guindo LA, Wagner M, Baltussen R, et al. From efficacy to equity: Literature review of decision criteria for resource allocation and healthcare decisionmaking. Cost Eff Resour Alloc. 2012; 10(9):1-13.

40. Kaur G, Prinja S, Lakshmi PVM, et al. Criteria Used for Priority-Setting for Public Health Resource Allocation in Low- and Middle-Income Countries: A Systematic Review. Int J Technol Assess Health Care. 2019; 35(6):474-483.

41. Jakovljevic MB. Resource allocation strategies in Southeastern European health policy. Eur J Health Econ. 2013; 14:153-159.

42. Fraser KD, Estabrooks C. What Factors Influence Case Managers' Resource Allocation Decisions? A 
Systematic Review of the Literature. Medical Decision Making. 2008; 12:394-410.

43. Haghparast-Bidgoli H, Kiadaliri AA, Skordis-Worrall J. Do economic evaluation studies inform effective healthcare resource allocation in Iran? A critical review of the literature. Cost Eff Resour Alloc. 2014; 12(15):1-11.

44. Craig AP, Thein HH, Zhang L, et al. Spending of HIV resources in Asia and Eastern Europe: systematic review reveals the need to shift funding allocations towards priority populations. J Int AIDS Soc. 2014; 25(17):18822.

45. Pinho MM, Veiga PACV. Avaliação de custo-utilidade como mecanismo de alocação de recursos em saúde: revisão do debate. Cad. Saúde Pública. 2009; 25(2):239-250.

46. Steuten L, Buxton M. Economic evaluation of healthcare safety: which attributes of safety do healthcare professionals consider most important in resource allocation decisions? Qual Saf Health Care. 2010 [acesso em 2019 nov 1]; 19(5):e6. Disponível em: https://qualitysafety.bmj.com/content/qhc/early/2010/08/10/qshc.2008.027870.full.pdf.
47. Anselmi L, Lagarde M, Hanson K. Equity in the allocation of public sector financial resources in low- and middle-income countries: a systematic literature review. Health Policy Plan. 2015; 30:528-545.

48. Mendes A, Carnut L, Guerra LDS. Reflexões acerca do financiamento federal da Atenção Básica no Sistema Único de Saúde. Saúde debate. 2018; 42(esp):224243.

49. Vianna SM, Piola SF, Guerra AJ, et al. O financiamento da descentralização dos serviços de saúde: critérios para transferências de recursos federais para estados e municípios. Brasília, DF: Opas, 1991.

50. Anhaia S, Almeida S, Santos R, et al. Challenges in the allocation of resources in health: an ethical approach. Rev. enferm. UFPE. 2011; 5(3):836-842.

Recebido em 19/10/2019

Aprovado em 30/04/2020

Conflito de interesses: inexistente

Suporte financeiro: Ministério da Saúde 